\title{
Sex, Age, and Race Effects on Immunogenicity of MenB-FHbp, A Bivalent Meningococcal B Vaccine: Pooled Evaluation of Clinical Trial Data
}

\author{
Johannes Beeslaar · Paula Peyrani · Judith Absalon · Jason Maguire • \\ Joseph Eiden · Paul Balmer · Roger Maansson · John L. Perez
}

Received: April 8, 2020 / Published online: July 17, 2020

(C) Pfizer Inc. 2020

\begin{abstract}
Introduction: An extensive clinical development program showed that the meningococcal serogroup B-factor $\mathrm{H}$ binding protein (MenBFHbp) vaccine affords protection against MenB disease for adolescents and adults. Data were pooled from multiple studies within the program to examine whether MenB-FHbp immunogenicity was influenced by sex, age, or race.
\end{abstract}

Methods: Immunogenicity was assessed in subjects from seven studies who received $120 \mu \mathrm{g}$ MenB-FHbp (at 0, 2, 6 months) and had evaluated immune responses against four representative test strains via serum bactericidal assays

Digital Features To view digital features for this article go to https://doi.org/10.6084/m9.figshare.12504986.

J. Beeslaar $(\square)$

Pfizer Vaccine Clinical Research and Development, Hurley, UK

e-mail: johannes.beeslaar@pfizer.com

P. Peyrani · R. Maansson · J. L. Perez

Pfizer Vaccine Clinical Research and Development, Collegeville, PA, USA

J. Absalon · J. Maguire · J. Eiden

Pfizer Vaccine Clinical Research and Development, Pearl River, NY, USA

P. Balmer

Pfizer Vaccine Medical Development, Scientific and Clinical Affairs, Collegeville, PA, USA using human complement (hSBAs). Immune responses were presented by sex (male, female), age group (10-14, 15-18, 19-25, 10-25 years), and race (white, black, Asian, other).

Results: Among 8026 subjects aged 10-25 years included in this analysis, MenB-FHbp elicited robust immune responses in a high percentage of subjects regardless of demographic characteristics. Across all test strains and demographic subsets, a $\geq 4$-fold rise in titer from baseline was achieved in 76.7-95.0\% of subjects, with no major differences by sex, age groups assessed, or races evaluated. Corresponding percentages achieving titers $\geq$ the lower limit of quantification (LLOQ) against all four strains combined were $79.7-87.3 \%$ (sex), 81.6-85.5\% (age), and $80.0-88.1 \%$ (race). Minor differences were observed for geometric mean titers and percentages of subjects achieving titers $\geq$ LLOQ against each strain based on demographics.

Conclusion: These data suggested no clinically meaningful differences in MenB-FHbp immunogenicity when administered as a threedose schedule based on sex, ages assessed, or races evaluated. This analysis supports the continued recommended use of MenB-FHbp to prevent MenB disease in adolescents and young adults.

Trial Registration: ClinicalTrials.gov identifiers, NCT00808028, NCT01830855, NCT01323270, NCT01461993, NCT01461980, NCT01352845, and NCT01299480. 
Keywords: Clinical trial; Immunogenicity; Invasive meningococcal disease; Serogroup B; Vaccines

\section{Key Summary Points}

\section{Why carry out this study?}

MenB-FHbp is a vaccine for the prevention of meningococcal disease caused by meningococcal serogroup B (MenB), which predominates in many global regions.

Although an extensive clinical program supported MenB-FHbp immunogenicity, influences of demographic characteristics on immune responses have not been evaluated.

Effects of sex, age, and race on immune responses induced by MenB-FHbp were evaluated using pooled data from seven randomized clinical studies in which adolescents or young adults received $120 \mu \mathrm{g}$ MenB-FHbp on a 0-, 2-, and 6-month schedule.

\section{What was learned from the study?}

MenB-FHbp was associated with robust immune responses against four diverse, vaccine-heterologous MenB test strains, with no clinically meaningful differences observed across demographic subgroups.

These findings confirm a three-dose MenBFHbp schedule can be used in adolescents and young adults regardless of sex, ages assessed, or races evaluated.

\section{INTRODUCTION}

Invasive meningococcal disease (IMD) is a rare but life-threatening condition caused by Neisseria meningitidis and is most commonly diagnosed in children aged $<1$ year, adolescents, and young adults [1]. Disease typically manifests as meningitis or bacteremia, with case fatality rates from $10 \%$ to $20 \%$ and debilitating long-term sequelae in up to $20 \%$ of survivors [1]. In several regions worldwide, meningococcal serogroup B (MenB) accounts for more cases of IMD than any other serogroup, causing 38\% of cases in the USA and $51 \%$ of cases in the European Union in 2017 [2, 3]. Vaccination is the most effective method for large-scale prevention of IMD [4].

MenB-FHbp (Trumenba ${ }^{\circledR}$, bivalent rLP2086; Pfizer Inc, Philadelphia, PA, USA), which consists of two recombinant factor $\mathrm{H}$ binding protein (FHbp) variants from each subfamily [subfamily A (variant A05) and subfamily B (variant B01)], is one of two vaccines licensed to prevent MenB disease [5, 6]. Because individual MenB strains generally express a single subfamily variant $[7,8]$, the MenB-FHbp formulation is predicted to broadly protect against diverse MenB disease-causing strains [7]. An extensive clinical development program has been completed for MenB-FHbp [9], but the impact of sex, age, and race on MenB-FHbp immunogenicity has not been systematically assessed.

Variability in vaccine responses based on age [10-14], sex [15-19], and race [20-23] has been observed for other vaccines. Therefore, it is important for public health authorities and medical practitioners to understand whether differences in vaccine efficacy occur in various populations in order to maximize vaccine benefit $[15,16,18,24]$. Moreover, understanding the challenges to effective vaccination in specific populations can inform vaccine clinical trial design and vaccine uptake after licensure $[18,20]$.

MenB-FHbp was shown to elicit robust immune responses and to have an acceptable safety profile during an extensive clinical development program, including studies enrolling 20,803 adolescents and adults [9]. Although data analyzed by demographic subsets have not been published, differences in point estimates of immunogenicity end points based on sex and race were observed in some of the individual studies [8]. However, there were too few participants within each study to provide a comprehensive assessment of the impact of 
demographics on immunogenicity. Therefore, the current analysis pooled data from studies across the MenB-FHbp clinical development program to generate a larger sample size for evaluating whether sex, age, or race affected MenB-FHbp immunogenicity.

\section{METHODS}

\section{Data Sources}

The MenB-FHbp clinical development program consisted of 11 clinical studies enrolling adults and adolescents [9]. Seven of these 11 clinical studies (Table 1) [8, 25-29] in which subjects who received $120 \mu \mathrm{g}$ of MenB-FHbp on a 0-, 2-, and 6-month schedule and had available immunogenicity results for some or all of the four primary test strains (described below) were included in this subgroup analysis. All seven studies had randomized designs, and six of the seven studies included comparator groups receiving either saline or another vaccine.

The data in this article were derived from previously conducted studies; as such, this article does not describe any new studies with human participants or animals and no new ethical approvals were needed.

\section{Immunogenicity Evaluation}

Immune responses were evaluated by serum bactericidal assays using human complement (hSBA) against the four primary test strains used in each study [9]. These included two strains expressing FHbp subfamily A variants (PMB80 expressing variant A22, PMB2001 expressing variant A56) and two strains expressing FHbp subfamily B variants (PMB2948 expressing variant B24, PMB2707 expressing variant B44) [9], hereafter referred to as test strains A22, A56, B24, and B44. The five coprimary end points assessing responses 1 month after dose 3 were:

- Percentage of subjects achieving $a \geq 4$-fold rise in titer from baseline against each of the four test strains (criteria for $\mathrm{a} \geq 4$-fold rise in titer have been previously described $[8,30]$ ).
- Percentage of subjects achieving titers $\geq$ the lower limit of quantification (LLOQ) against all four primary test strains combined (i.e., composite response; $\geq 1: 8$ for the test strains expressing variants A56, B24, and B44 and $\geq 1: 16$ for the test strain expressing variant A22)

Additional end points evaluated included:

- Percentage of subjects achieving titers $\geq$ LLOQ against each test strain

- Geometric mean titers (GMTs) against each test strain

- $\quad$ Percentage of subjects achieving titers $\geq 1: 4$ (the established correlate of protection $[31,32])$ against each test strain

\section{Statistical Analyses}

Descriptive statistics are provided for all end points; no hypothesis testing was performed. Percentages are expressed with 95\% CIs calculated using the Clopper-Pearson method, and GMTs are expressed as back transformations of the mean logarithm of hSBA titers along with 95\% CIs based on Student's $t$-test distributions. Results were presented by sex (male or female), age group [10-25 (total), 10-14, 15-18, 10-18, or 19-25 years], and race (white, black, Asian, or other) for all end points. Analysis by ethnicity (non-Hispanic/non-Latino, Hispanic/Latino, or unknown) was not conducted because data by ethnicity were not collected in all studies.

\section{RESULTS}

\section{Demographics}

The evaluable immunogenicity population included 8026 subjects aged 10-25 years who were randomly assigned to receive $120 \mu \mathrm{g}$ MenB-FHbp on a 0-, 2-, and 6-month schedule; demographic characteristics are summarized in Table 2. Percentages of males and females included in the evaluable immunogenicity population were similar. Most subjects (80.7\%) were adolescents aged 10-18 years at the time of study entry, and $53.5 \%$ of subjects were aged 


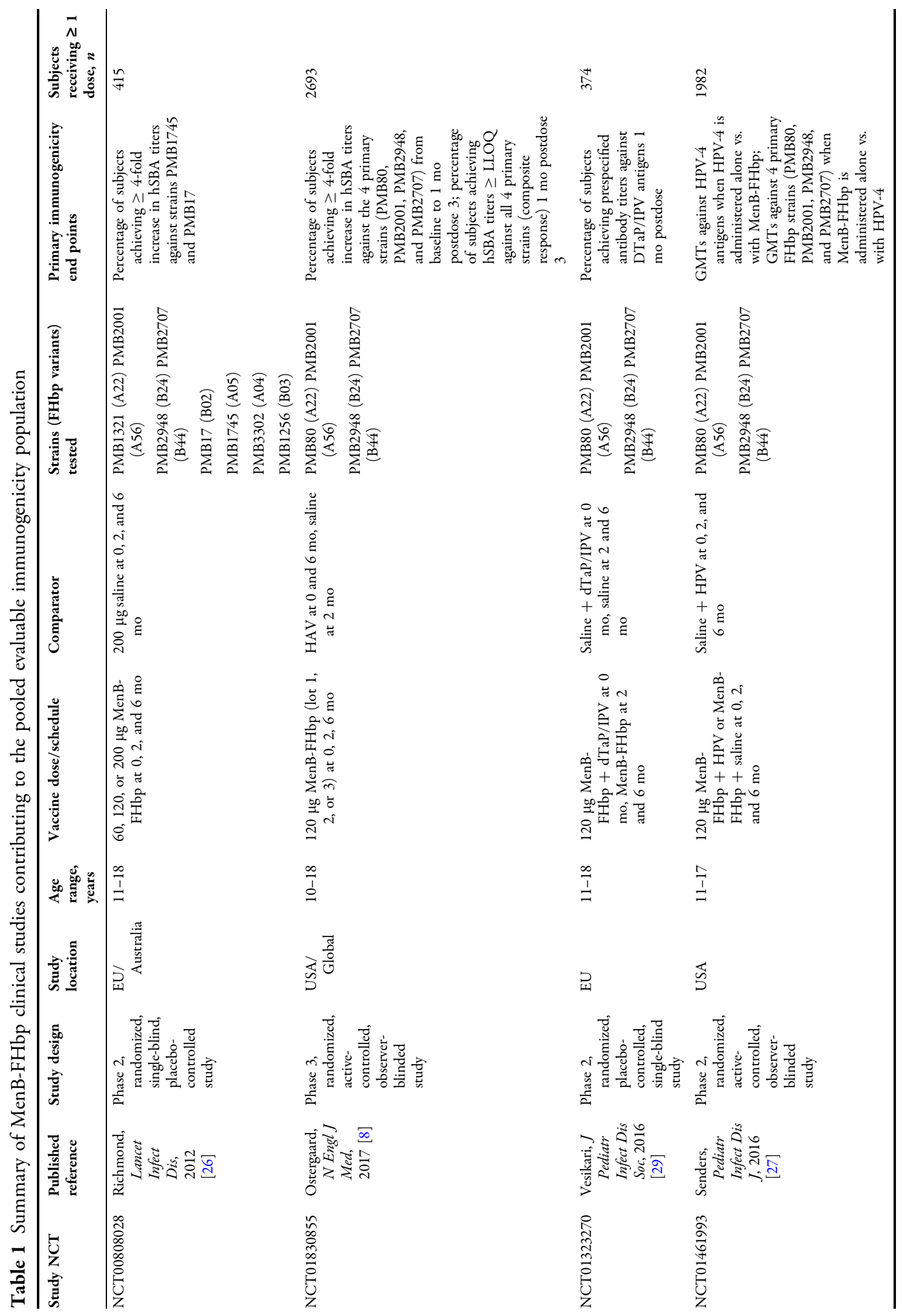




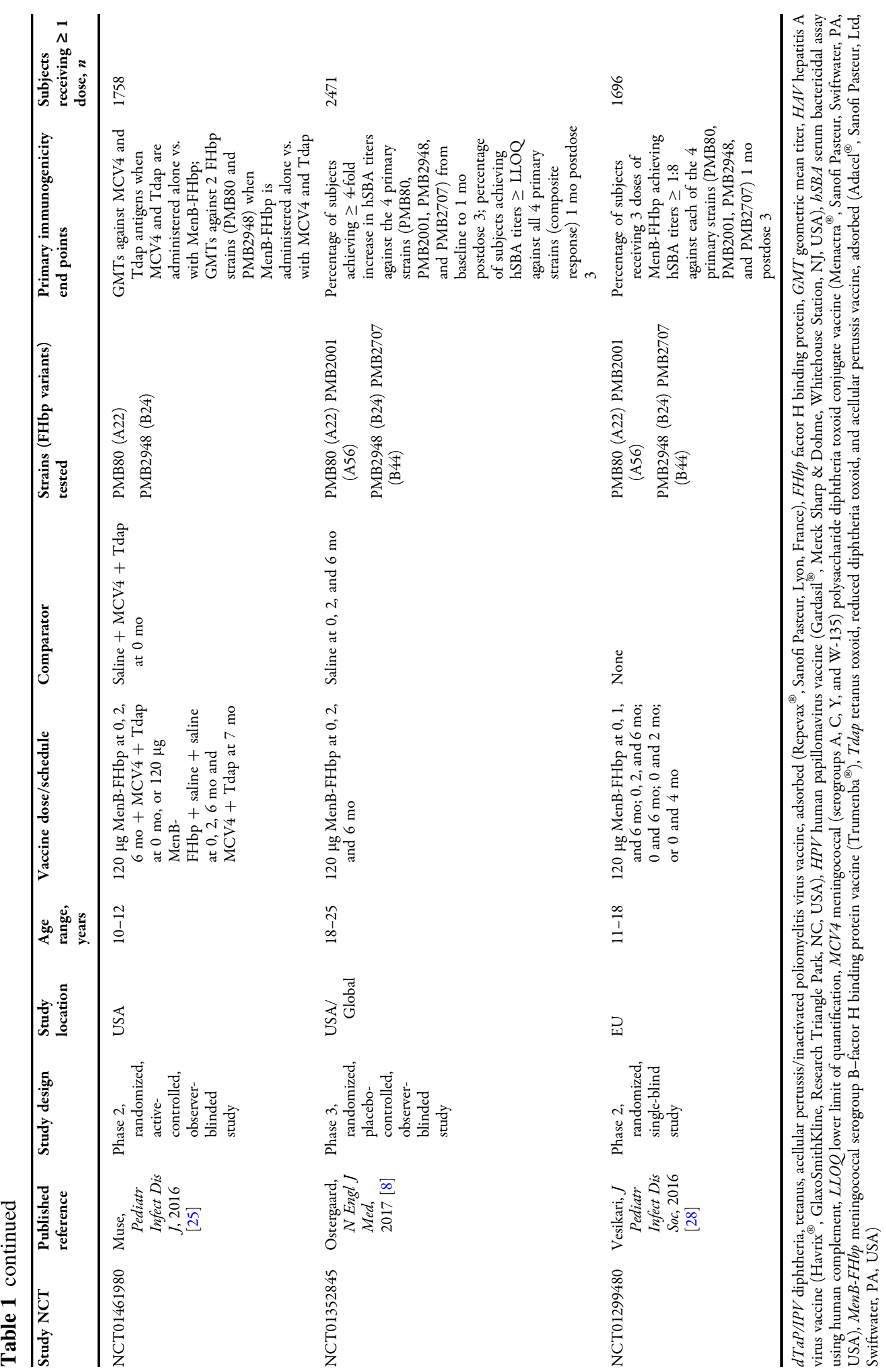


Table 2 Subject demographics in the pooled evaluable immunogenicity population

\begin{tabular}{ll}
\hline Total, $N$ & 8026 \\
Sex, $n$ (\%) & \\
Male & $4153(51.7)$ \\
Female & $3873(48.3)$ \\
Age group at first dose, years (\%) & \\
$10-18$ & $6474(80.7)$ \\
$10-14$ & $4290(53.5)$ \\
$15-18$ & $2184(27.2)$ \\
$19-25$ & $1552(19.3)$ \\
Age at first dose, years & \\
Mean \pm SD & $15.0 \pm 4.2$ \\
Median (range) & $14(10-25)$ \\
Race, $n$ (\%) & \\
White & $6982(87.0)$ \\
Black & $745(9.3)$ \\
Asian & $61(0.8)$ \\
Other & $238(3.0)$ \\
\hline
\end{tabular}

10-14 years. Most subjects (87.0\%) were white, with the remainder identifying as black $(9.3 \%)$, Asian (0.8\%), or other (3.0\%).

\section{Immunogenicity}

The percentage of subjects achieving a $\geq 4$-fold rise from baseline for each of the four test strains and percentages of subjects achieving a composite response 1 month after dose 3 are shown in Table 3. Regardless of demographics, high percentages of subjects achieved either a $\geq 4$-fold rise in titer from baseline for each test strain or a composite response.

For sex, the observed differences between percentages of males and females achieving a $\geq 4$-fold rise in titer from baseline were $<5 \%$ for test strains A22, A56, and B24, with males showing a greater response. For test strain B44, the percentage of males achieving $\mathrm{a} \geq 4$-fold rise in titer was $8.3 \%$ higher compared with females $(85.1 \%$ vs. $76.8 \%$, respectively). For the composite response, a higher percentage of males achieved titers $\geq$ LLOQ for all four test strains combined (7.6\% higher than in females).

Comparisons across age subgroups indicated no major differences between groups for $\mathrm{a} \geq 4$ fold rise in titers from baseline. A higher percentage of subjects aged 10-14 years achieved $a \geq 4$-fold rise in titer compared with subjects aged 15-18 and 19-25 years for strains A22, A56, and B24 (Table 3), and the 95\% CIs suggest that younger adolescents may have slightly increased hSBA responses compared with young adults. Specifically, $1.7-5.9 \%$ more subjects aged $10-14$ years showed $a \geq 4$-fold rise in titer from baseline than those aged 15-18 and 19-25 years, which was not considered clinically significant. In addition, subjects aged 10-18 years showed slightly greater responses against test strains A22 and A56 than those aged 19-25 years, with $4.1 \%$ and $3.0 \%$ more subjects responding, respectively. For test strain B44, observed differences in the percentage of subjects responding were $<2.4 \%$ across age subgroups, with overlapping $95 \%$ CIs. For composite responses, the greatest difference between subgroups was observed between subjects aged 15-18 and 19-25 years (3.9\%); however, it should be noted that CIs overlapped across all subgroups.

With regard to race, percentages of subjects achieving a $\geq 4$-fold rise in titers from baseline were generally consistent among white, black, Asian, and other subgroups (Table 3 ). The greatest percentage difference was observed between whites and Asians for test strain B24, for which $12.4 \%$ more Asian subjects achieved $\mathrm{a} \geq 4$-fold rise in titer from baseline compared with white subjects. However, it should be noted that these analyses are limited by the very low number of Asian subjects included ( $n=42-58$ per strain).

The percentage of subjects achieving titers $\geq$ LLOQ for each of the four test strains at 1 month after dose 3 is shown in Table 4 . Notably, percentages of subjects achieving titers $\geq$ LLOQ were high across all demographic groups, with only minor differences observed 


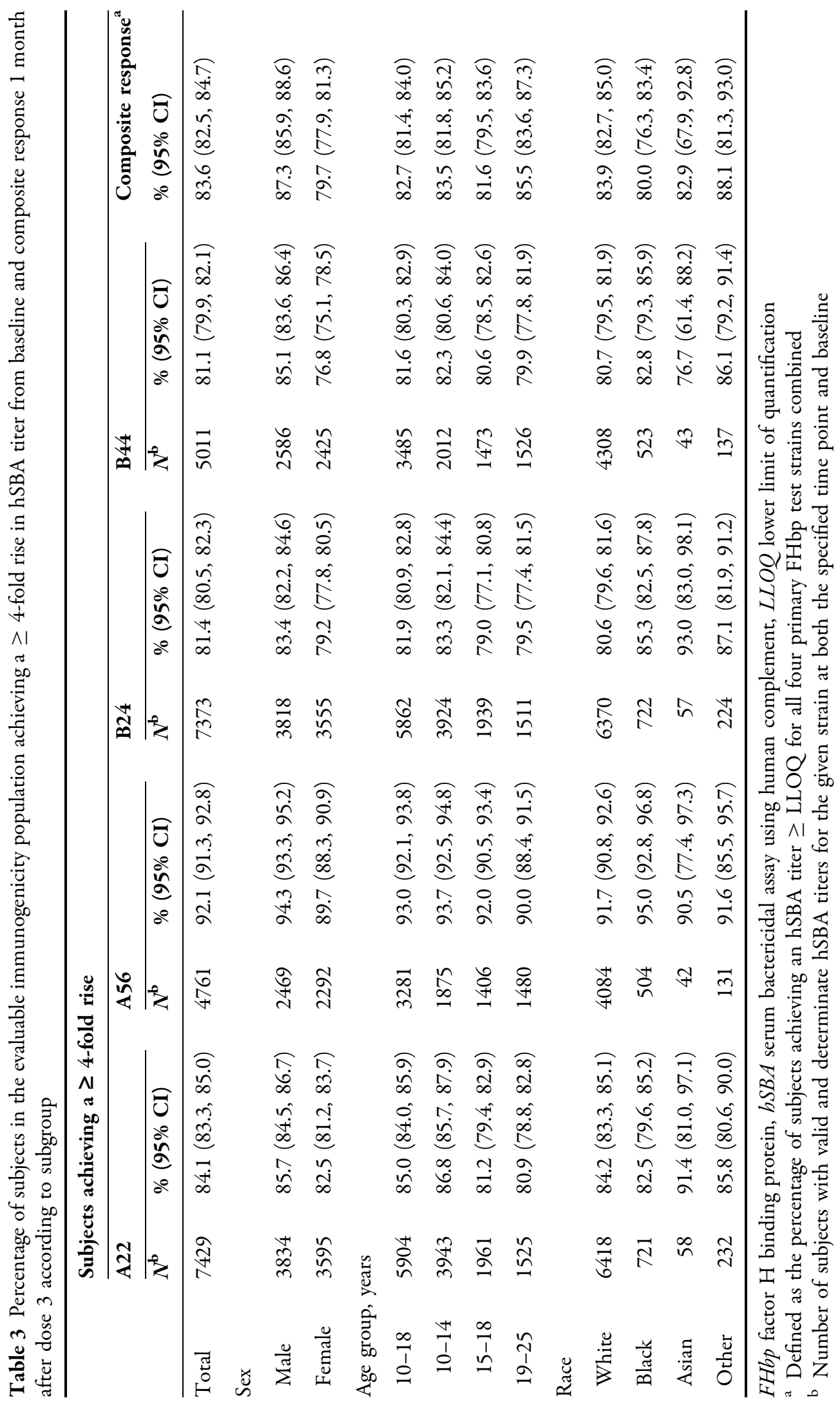


Table 4 Percentage of subjects in the evaluable immunogenicity population achieving hSBA titers $\geq$ LLOQ 1 month after dose 3 according to subgroup

\begin{tabular}{|c|c|c|c|c|c|c|c|c|}
\hline & \multicolumn{8}{|c|}{ Subjects with hSBA titers $\geq \operatorname{LLOQ}^{a}$} \\
\hline & \multicolumn{2}{|l|}{ A22 } & \multicolumn{2}{|l|}{ A56 } & \multicolumn{2}{|l|}{ B24 } & \multicolumn{2}{|l|}{ B44 } \\
\hline & $\overline{N^{\mathbf{b}}}$ & $\%(95 \% \mathrm{CI})$ & $\overline{N^{\mathbf{b}}}$ & $\%(95 \% \mathrm{CI})$ & $N^{\mathbf{b}}$ & $\%(95 \% \mathrm{CI})$ & $\overline{N^{\mathbf{b}}}$ & $\%(95 \% \mathrm{CI})$ \\
\hline Total & 7577 & $94.5(94.0,95.0)$ & 5084 & $99.3(99.1,99.5)$ & 7478 & $91.2(90.5,91.8)$ & 5046 & $86.6(85.7,87.6)$ \\
\hline \multicolumn{9}{|l|}{ Sex } \\
\hline Male & 3915 & $95.1(94.4,95.8)$ & 2620 & $99.4(99.0,99.7)$ & 3880 & $93.2(92.4,94.0)$ & 2609 & $89.9(88.7,91.0)$ \\
\hline Female & 3662 & $93.8(93.0,94.6)$ & 2464 & $99.3(98.8,99.6)$ & 3598 & $88.9(87.9,89.9)$ & 2437 & $83.2(81.6,84.6)$ \\
\hline \multicolumn{9}{|c|}{ Age group, years } \\
\hline $10-18$ & 6033 & $94.7(94.1,95.2)$ & 3547 & $99.3(98.9,99.5)$ & 5944 & $90.1(89.3,90.8)$ & 3513 & $86.1(84.9,87.2)$ \\
\hline $10-14$ & 4031 & $94.6(93.9,95.3)$ & 2038 & $99.5(99.1,99.8)$ & 3971 & $90.6(89.7,91.5)$ & 2024 & $86.5(84.9,88.0)$ \\
\hline $15-18$ & 2002 & $94.8(93.7,95.7)$ & 1509 & $98.9(98.3,99.4)$ & 1973 & $89.0(87.5,90.3)$ & 1489 & $85.4(83.5,87.2)$ \\
\hline $19-25$ & 1544 & $93.8(92.5,95.0)$ & 1537 & $99.5(99.0,99.8)$ & 1534 & $95.4(94.2,96.4)$ & 1533 & $88.0(86.3,89.6)$ \\
\hline \multicolumn{9}{|l|}{ Race } \\
\hline White & 6547 & $94.8(94.3,95.4)$ & 4366 & $99.3(99.0,99.5)$ & 6458 & $91.0(90.2,91.6)$ & 4339 & $86.7(85.7,87.7)$ \\
\hline Black & 736 & $91.6(89.3,93.5)$ & 534 & $99.6(98.7,100.0)$ & 733 & $92.1(89.9,93.9)$ & 527 & $85.6(82.3,88.5)$ \\
\hline Asian & 60 & $96.7(88.5,99.6)$ & 43 & $100.0(91.8,100.0)$ & 59 & $98.3(90.9,100.0)$ & 43 & $81.4(66.6,91.6)$ \\
\hline Other & 234 & $94.0(90.2,96.7)$ & 141 & $99.3(96.1,100.0)$ & 228 & $92.5(88.3,95.6)$ & 137 & $90.5(84.3,94.9)$ \\
\hline
\end{tabular}

between subgroups. For sex, observed differences in the percentage of males and females achieving titers $\geq$ LLOQ were $<5 \%$ between subgroups for all test strains except B44, for which the percent difference in response was $6.7 \%$ higher among males than females. Minor or no differences in the percentage of subjects with hSBA titers $\geq$ LLOQ were observed across age groups for test strains A22, A56, and B44. However, for test strain B24, a higher percentage of subjects aged 19-25 years achieved titers $\geq$ LLOQ compared with those aged 10-14, $10-18$, and $15-18$ years $(4.8 \%, 5.3 \%$, and $6.4 \%$ more respondent subjects, respectively). For race subgroups, minor between-group differences were observed for percentages with titers $\geq$ LLOQ against each test strain.
Geometric mean titers 1 month after dose 3 are shown in Table 5. Comparisons across sex, age, and race generally indicated only minor differences between subgroups. For sex, observed GMTs for all four test strains were slightly higher in males compared with females, with the greatest difference observed for test strain A56 (171.8 vs. 157.3, respectively). With regard to age, the 19- to 25-year-old age group showed higher observed GMTs against all four test strains than seen in the other age subgroups, with the greatest differences observed for test strains A56 and B24. Across races, minor differences in GMTs were observed for all four test strains.

The percentage of subjects achieving titers $\geq 1: 4$ across each of the four test strains after dose 3 is shown in Table 6 . Overall, only minor 
Table 5 hSBA GMTs in the evaluable immunogenicity population 1 month after dose 3 according to subgroup

\begin{tabular}{|c|c|c|c|c|c|c|c|c|}
\hline & \multicolumn{8}{|c|}{ hSBA GMT } \\
\hline & \multicolumn{2}{|c|}{ A22 } & \multicolumn{2}{|l|}{ A56 } & \multicolumn{2}{|l|}{ B24 } & \multicolumn{2}{|l|}{ B44 } \\
\hline & $\overline{N^{a}}$ & GMT (95\% CI) & $\overline{N^{a}}$ & GMT (95\% CI) & $\overline{N^{a}}$ & GMT (95\% CI) & $\overline{N^{a}}$ & GMT $(95 \% \mathrm{CI})$ \\
\hline Total & 7577 & $66.5(65.0,68.1)$ & 5084 & $164.6(160.0,169.3)$ & 7478 & $30.0(29.3,30.8)$ & 5046 & $40.4(38.9,42.1)$ \\
\hline \multicolumn{9}{|l|}{ Sex } \\
\hline Male & 3915 & $70.0(67.8,72.3)$ & 2620 & $171.8(165.3,178.7)$ & 3880 & $32.0(31.0,33.1)$ & 2609 & $45.0(42.7,47.4)$ \\
\hline Female & 3662 & $63.0(60.9,65.1)$ & 2464 & $157.3(151.0,163.8)$ & 3598 & $28.0(27.0,29.0)$ & 2437 & $36.1(34.0,38.3)$ \\
\hline \multicolumn{9}{|c|}{ Age group, years } \\
\hline $10-18$ & 6033 & $64.3(62.8,65.9)$ & 3547 & $159.0(153.8,164.3)$ & 5944 & $26.2(25.5,26.9)$ & 3513 & $37.1(35.5,38.8)$ \\
\hline $10-14$ & 4031 & $62.4(60.6,64.2)$ & 2038 & $161.9(155.4,168.7)$ & 3971 & $25.4(24.6,26.1)$ & 2024 & $37.3(35.2,39.5)$ \\
\hline $15-18$ & 2002 & $68.4(65.3,71.5)$ & 1509 & $155.1(147.0,163.6)$ & 1973 & $28.0(26.7,29.3)$ & 1489 & $36.9(34.4,39.5)$ \\
\hline $19-25$ & 1544 & $75.9(71.6,80.5)$ & 1537 & $178.4(169.0,188.3)$ & 1534 & $50.9(47.9,54.0)$ & 1533 & $49.3(45.6,53.3)$ \\
\hline \multicolumn{9}{|l|}{ Race } \\
\hline White & 6547 & $67.7(66.0,69.4)$ & 4366 & $166.5(161.4,171.7)$ & 6458 & $30.0(29.2,30.8)$ & 4339 & $41.0(39.3,42.8)$ \\
\hline Black & 736 & $55.5(51.6,59.7)$ & 534 & $144.8(134.2,156.2)$ & 733 & $29.0(27.1,31.1)$ & 527 & $35.0(31.3,39.2)$ \\
\hline Asian & 60 & $67.0(53.1,84.6)$ & 43 & $165.7(129.5,212.0)$ & 59 & $34.3(26.9,43.8)$ & 43 & $33.0(20.8,52.4)$ \\
\hline Other & 234 & $72.7(64.3,82.1)$ & 141 & $187.8(159.5,221.2)$ & 228 & $31.9(27.8,36.6)$ & 137 & $48.5(37.7,62.4)$ \\
\hline
\end{tabular}

$G M T$ geometric mean titer, $h S B A$ serum bactericidal assay using human complement

${ }^{a}$ Number of subjects with valid and determinate hSBA titers for the given strain

differences in the percentage of subjects achieving titers $\geq 1: 4$ were observed by sex, age, and race across each of the four test strains.

\section{DISCUSSION}

Robust immunogenicity of MenB-FHbp has been individually shown in each of the 11 clinical studies collectively enrolling $>20,000$ adolescents and adults [9]. A pooled analysis from seven of these clinical studies, which included $>8000$ subjects from the clinical development program, was conducted to detect any emergent effects of demographic factors on MenB-FHbp immunogenicity. In this subgroup analysis, immunogenicity was similar across sex, ages assessed, and race (predominantly white and black). Some variation in hSBA responses was observed between individual test strains across all groups analyzed; this is expected based on differences in FHbp sequences and cell surface expression levels across MenB strains [33, 34]. Moreover, although minor differences by sex were observed among percentages of subjects with $\geq 4$-fold rise in titers from baseline, percentages of subjects with titers $\geq$ LLOQ, and GMTs, the percentages of subjects with titers $\geq 1: 4$ against each test strain were highly consistent. Because an hSBA titer of $1: 4$ is the accepted correlate of protection from meningococcal disease [31, 32], the slight differences in immunogenicity end points reported here are unlikely to be clinically meaningful and do not impact current MenBFHbp vaccination recommendations [5]. Although the current analysis was focused on immunogenicity only, it should be noted that a 
Table 6 Percentage of subjects in the evaluable immunogenicity population achieving hSBA titers $\geq 1: 41$ month after dose 3 according to subgroup

\begin{tabular}{|c|c|c|c|c|c|c|c|c|}
\hline & \multicolumn{8}{|c|}{ Subjects with hSBA titers $\geq 1: 4$} \\
\hline & \multicolumn{2}{|l|}{$\overline{A 22}$} & \multicolumn{2}{|l|}{ A56 } & \multicolumn{2}{|l|}{ B24 } & \multicolumn{2}{|l|}{ B44 } \\
\hline & $\overline{N^{\mathbf{a}}}$ & $\%(95 \% \mathrm{CI})$ & $\overline{N^{a}}$ & $\%(95 \% \mathrm{CI})$ & $\overline{N^{a}}$ & $\%(95 \% \mathrm{CI})$ & $\overline{N^{a}}$ & $\%(95 \% \mathrm{CI})$ \\
\hline Total & 7577 & $94.9(94.4,95.4)$ & 5084 & $99.4(99.1,99.6)$ & 7478 & $92.2(91.6,92.8)$ & 5046 & $88.2(87.3,89.1)$ \\
\hline \multicolumn{9}{|l|}{ Sex } \\
\hline Male & 3915 & $95.6(94.9,96.2)$ & 2620 & $99.5(99.1,99.7)$ & 3880 & $94.4(93.7,95.1)$ & 2609 & $91.1(89.9,92.2)$ \\
\hline Female & 3662 & $94.1(93.3,94.9)$ & 2464 & $99.3(98.9,99.6)$ & 3598 & $89.9(88.9,90.8)$ & 2437 & $85.1(83.7,86.5)$ \\
\hline \multicolumn{9}{|c|}{ Age group, years } \\
\hline $10-18$ & 6033 & $95.0(94.4,95.5)$ & 3547 & $99.4(99.0,99.6)$ & 5944 & $91.3(90.5,92.0)$ & 3513 & $87.3(86.2,88.4)$ \\
\hline $10-14$ & 4031 & $94.9(94.1,95.5)$ & 2038 & $99.5(99.1,99.8)$ & 3971 & $91.6(90.7,92.5)$ & 2024 & $87.9(86.4,89.3)$ \\
\hline $15-18$ & 2002 & $95.2(94.2,96.1)$ & 1509 & $99.1(98.5,99.5)$ & 1973 & $90.5(89.1,91.8)$ & 1489 & $86.6(84.7,88.3)$ \\
\hline $19-25$ & 1544 & $94.6(93.3,95.6)$ & 1537 & $99.5(99.0,99.8)$ & 1534 & $96.1(95.0,97.0)$ & 1533 & $90.3(88.7,91.7)$ \\
\hline \multicolumn{9}{|l|}{ Race } \\
\hline White & 6547 & $95.2(94.7,95.7)$ & 4366 & $99.4(99.1,99.6)$ & 6458 & $92.1(91.4,92.8)$ & 4339 & $88.4(87.4,89.4)$ \\
\hline Black & 736 & $92.1(89.9,94.0)$ & 534 & $99.6(98.7,100.0)$ & 733 & $92.6(90.5,94.4)$ & 527 & $86.0(82.7,88.8)$ \\
\hline Asian & 60 & $96.7(88.5,99.6)$ & 43 & $100.0(91.8,100.0)$ & 59 & $98.3(90.9,100.0)$ & 43 & $83.7(69.3,93.2)$ \\
\hline Other & 234 & $94.4(90.7,97.0)$ & 141 & $99.3(96.1,100.0)$ & 228 & $93.0(88.9,95.9)$ & 137 & $92.0(86.1,95.9)$ \\
\hline
\end{tabular}

hSBA serum bactericidal assay using human complement

${ }^{a}$ Number of subjects with valid and determinate hSBA titers for the given strain

previous analysis identified no safety concerns among data pooled from adolescents and adults in the MenB-FHbp clinical development program (unpublished data).

Sex, age, and race were selected as factors to include in this analysis because these characteristics have previously been associated with variable immunogenicity of numerous vaccines [10-12, 15-23]. These demographic influences also interact with one another, illustrated in part by fluctuations in sex-dependent immune responses at different times throughout the lifespan [15, 19, 24]. A commonly observed influential variable is age, which is typically associated with decreased immunogenicity in infants and adults aged $\geq 65$ years $[10,11]$. As an example, clinical trials with live-attenuated and inactivated influenza vaccines are of interest to highlight differences in vaccine efficacy by age group. In a study of children aged 6-59 months, there were $54.9 \%$ fewer cases of culture-confirmed influenza among study children who received the live-attenuated vaccine compared with the group who received the inactivated vaccine [35]. In contrast, in a study of adults aged 18-49 years, the inactivated vaccine provided a $50 \%$ reduction in confirmed influenza compared with those given the liveattenuated vaccine [36]. Among children, adolescents, and young adults, aging may either increase or decrease immune responses depending on the vaccine [37-40]. Variable vaccine efficacy among the above-listed age groups has also been shown specifically for some strain-specific MenB outer membrane vesicle (OMV) vaccines. For example, a case- 
control study of an OMV antigen-based vaccine developed in Cuba found that protective efficacy was greater among Brazilian adults aged 24-47 years compared with those aged < 24 years [39]. In a review of OMV antigen-based MenB vaccines with vaccine efficacy or hSBA data published before 2015, several studies showed variability across age groups in both vaccine effectiveness and hSBA responses [40]. Additionally, there is evidence to suggest immune responses to vaccines can vary even within the specific age range included in this study. Two studies investigating the immunogenicity of a bivalent human papillomavirus vaccine among female subjects aged 10-25 years found that antibody responses following vaccination were higher in subjects aged 10-14 years compared with those aged 15-25 years [13, 14]. However, in the study presented here, the small differences observed in immune responses between different age groups were not considered clinically meaningful.

Sex differences in vaccine response have also been reported for some vaccines [15-19]. Although the reasons for these differences are not fully understood [15], underlying mechanisms may include sex-based differences in innate and adaptive immunity $[17,18]$, modulations of immune cell function by sex hormones [15-17, 24], genetic influences $[15,17,24]$, and sex-specific features of the diet or microbiome [15]. Most vaccines elicit stronger immune responses in women than in men $[15,17]$, and this trend persists throughout the lifespan from infancy into late adulthood $[19,24]$. An example of this observation is the seasonal trivalent inactivated influenza vaccine, which produces significantly higher GMTs in women compared with men, regardless of dose amount or age [41].

Additionally, some vaccines are associated with variable immune responses based on race or geographic location of the vaccinated subject [20-23, 42, 43]. For example, compared with infants from other countries, those from the Philippines showed lower seroprotection rates and geometric mean concentrations against hepatitis B after vaccination with the combined diphtheria, tetanus, and whole cell pertussis-hepatitis B virus and Haemophilus influenzae type $\mathrm{b}$ conjugate vaccine with oral live attenuated poliovirus vaccine (DTPw-HBV/Hib-TT + OPV) coadministered with either a 7 - or 10 -valent pneumococcal conjugate vaccine [43]. These and other variable responses could result from genetic or environmental factors, although the precise contributions of each are unclear [20-23]. Because the predominant disease-causing vaccine serotypes may differ across global regions $[44,45]$, responses to vaccinations may also differ geographically.

The effects of sex, age, and race have not been comprehensively reported for licensed, broad-spectrum MenB vaccines. Controlled studies that investigate the possible influence of demographics on vaccine immunogenicity are important for informing vaccine recommendations $[15,16,19,24]$. The main strength of this subgroup analysis was its large sample size of $>8000$ subjects from the pooled evaluable immunogenicity population across seven randomized clinical studies conducted with similar methodology. Limitations include the relatively restricted age range of subjects (10-25 years), although forthcoming data could potentially address subgroup differences in immunogenicity among younger cohorts. Moreover, generalizability of age and race results is limited by the age groups and races included in this study, as most of the evaluable population was aged 10-18 years $(80.7 \%)$ and white $(87.0 \%)$. Additionally, most of the racial diversity from this analysis came from only a small number of studies; most of the subjects included were white or black, with Asians and other races representing $0.8 \%$ and $3 \%$ of the total evaluable immunogenicity population, respectively. As such, studies including a greater number of Asians and other races are needed to reach meaningful conclusions regarding immune responses to MenB-FHbp in these groups. Furthermore, this study focuses on the three-dose MenB-FHbp vaccination schedule; the effects of sex, age, and race on immune responses following a two-dose (0-, 6-month) schedule, which is also approved [5], were not evaluated. Despite these considerations, it is notable that these analyses suggested no clinically meaningful differences in immunogenicity between groups based on sex, ages assessed, or races 
evaluated, which indicates that no changes in the clinical management of these subpopulations are warranted regarding vaccination with MenB-FHbp. Additional insight could be provided by long-term follow-up from studies further evaluating the impact of demographics on immunogenicity.

\section{CONCLUSIONS}

The immunogenicity profile of the MenB-FHbp vaccine suggested that there were no clinically meaningful differences by sex, age groups assessed or race (predominantly white and black) across three-dose data pooled from the clinical development program. The robust immune responses and lack of clinically significant differences across demographic groups support the continued use of MenB-FHbp for prevention of MenB disease in these populations, as is currently recommended by health authorities.

\section{ACKNOWLEDGEMENTS}

Funding. This work was sponsored by Pfizer Inc. The Rapid Service Fees of Infectious Diseases and Therapy have been funded by Pfizer Inc.

Authorship. All named authors had full access to all of the data in this study, meet the International Committee of Medical Journal Editors (ICMJE) criteria for authorship for this article, take responsibility for the integrity of the work as a whole, and have given their approval for this version to be published.

Medical Writing, Editorial, and Other Assistance. Editorial/medical writing support was provided by Anna L. Stern, PhD, of ICON plc (North Wales, PA, USA) and was funded by Pfizer Inc.

Prior Presentation. This manuscript is based on work that was previously presented at the
IDWeek 2019 conference that took place in Washington, DC, USA, on October 2-6, 2019.

Disclosures. Johannes Beeslaar, Paula Peyrani, Judith Absalon, Jason Maguire, Joseph Eiden, Paul Balmer, Roger Maansson, and John L. Perez are current or former employees of Pfizer Inc. and may hold stock or stock options. Joseph Eiden was an employee of Pfizer Inc and is currently is retired.

Compliance with Ethics Guidelines. The data in this article were derived from previously conducted studies; as such, this article does not describe any new studies with human participants or animals performed, and no new ethical approvals were needed.

Data Availability. Upon request, and subject to certain criteria, conditions, and exceptions (see https://www.pfizer.com/science/ clinical-trials/trial-data-and-results for more information), Pfizer will provide access to individual de-identified participant data from Pfizer-sponsored global interventional clinical studies conducted for medicines, vaccines, and medical devices (1) for indications that have been approved in the US and/or EU or (2) in programs that have been terminated (i.e., development for all indications has been discontinued). Pfizer will also consider requests for the protocol, data dictionary, and statistical analysis plan. Data may be requested from Pfizer trials 24 months after study completion. The deidentified participant data will be made available to researchers whose proposals meet the research criteria and other conditions, and for which an exception does not apply, via a secure portal. To gain access, data requestors must enter into a data access agreement with Pfizer.

Open Access. This article is licensed under a Creative Commons Attribution-NonCommercial 4.0 International License, which permits any non-commercial use, sharing, adaptation, distribution and reproduction in any medium or format, as long as you give appropriate credit to the original author(s) and the source, provide a link to the Creative Commons licence, and indicate if changes were made. The images or 
other third party material in this article are included in the article's Creative Commons licence, unless indicated otherwise in a credit line to the material. If material is not included in the article's Creative Commons licence and your intended use is not permitted by statutory regulation or exceeds the permitted use, you will need to obtain permission directly from the copyright holder. To view a copy of this licence, visit http://creativecommons.org/licenses/by$\mathrm{nc} / 4.0 /$.

\section{REFERENCES}

1. Martinón-Torres F. Deciphering the burden of meningococcal disease: conventional and underrecognized elements. J Adolesc Health. 2016;59: S12-20.

2. Centers for Disease Control and Prevention. Enhanced meningococcal disease surveillance report (2017). https://www.cdc.gov/ meningococcal/downloads/NCIRD-EMS-Report2017.pdf.

3. European Centre for Disease Prevention and Control. Surveillance Atlas of Infectious Diseases (2019). https://ecdc.europa.eu/en/surveillance-atlasinfectious-diseases. Accessed 3 June 2019.

4. Balmer P, York LJ. Optimal use of meningococcal serogroup B vaccines: moving beyond outbreak control. Ther Adv Vaccines Immunother. 2018;6: 49-60.

5. Trumenba $^{\circledR}$ (meningococcal group B vaccine). Full Prescribing Information, Wyeth Pharmaceuticals Inc (a subsidiary of Pfizer Inc), Philadelphia; 2018.

6. Bexsero $^{\circledR}$ (meningococcal group B vaccine). Full Prescribing Information, GSK Vaccines, Srl, Siena; 2018.

7. Murphy E, Andrew L, Lee KL, et al. Sequence diversity of the factor $\mathrm{H}$ binding protein vaccine candidate in epidemiologically relevant strains of serogroup B Neisseria meningitidis. J Infect Dis. 2009;200:379-89.

8. Ostergaard L, Vesikari T, Absalon J, et al. A bivalent meningococcal B vaccine in adolescents and young adults. N Engl J Med. 2017;347:2349-62.

9. Perez JL, Absalon J, Beeslaar J, et al. From research to licensure and beyond: clinical development of
MenB-FHbp, a broadly protective meningococcal B vaccine. Expert Rev Vaccines. 2018;17:461-77.

10. Lord JM. The effect of ageing of the immune system on vaccination responses. Hum Vaccines Immunother. 2013;9:1364-7.

11. Demirjian A, Levy O. Safety and efficacy of neonatal vaccination. Eur J Immunol. 2009;39:36-46.

12. van Werkhoven $\mathrm{CH}$, Huijts $\mathrm{SM}$, Bolkenbaas $\mathrm{M}$, Grobbee DE, Bonten MJ. The impact of age on the efficacy of 13-valent pneumococcal conjugate vaccine in elderly. Clin Infect Dis. 2015;61:1835-8.

13. Sow PS, Watson-Jones D, Kiviat N, et al. Safety and immunogenicity of human papillomavirus-16/18 AS04-adjuvanted vaccine: a randomized trial in 10-25-year-old HIV-seronegative African girls and young women. J Infect Dis. 2013;207:1753-63.

14. Pedersen C, Petaja T, Strauss G, et al. Immunization of early adolescent females with human papillomavirus type 16 and $18 \mathrm{~L} 1$ virus-like particle vaccine containing AS04 adjuvant. J Adolesc Health. 2007;40:564-71.

15. Flanagan KL, Fink AL, Plebanski M, Klein SL. Sex and gender differences in the outcomes of vaccination over the life course. Annu Rev Cell Dev Biol. 2017;33:577-99.

16. Jorgensen TN. Sex disparities in the immune response. Cell Immunol. 2015;294:61-2.

17. Klein SL, Jedlicka A, Pekosz A. The Xs and Y of immune responses to viral vaccines. Lancet Infect Dis. 2010;10:338-49.

18. Klein SL, Marriott I, Fish EN. Sex-based differences in immune function and responses to vaccination. Trans R Soc Trop Med Hyg. 2015;109:9-15.

19. Fink AL, Klein SL. Sex and gender impact immune responses to vaccines among the elderly. Physiology (Bethesda). 2015;30:408-16.

20. Haralambieva IH, Salk HM, Lambert ND, et al. Associations between race, sex and immune response variations to rubella vaccination in two independent cohorts. Vaccine. 2014;32:1946-53.

21. Kurupati R, Kossenkov A, Haut L, et al. Race-related differences in antibody responses to the inactivated influenza vaccine are linked to distinct pre-vaccination gene expression profiles in blood. Oncotarget. 2016;7:62898-911.

22. Moberley S, Licciardi PV, Balloch A, et al. Repeat pneumococcal polysaccharide vaccine in Indigenous Australian adults is associated with decreased 
immune responsiveness. Vaccine. 2017;35: 2908-15.

23. Rager-Zisman B, Bazarsky E, Skibin A, et al. Differential immune responses to primary measlesmumps-rubella vaccination in Israeli children. Clin Diagn Lab Immunol. 2004;11:913-8.

24. Voysey M, Barker CI, Snape MD, et al. Sex-dependent immune responses to infant vaccination: an individual participant data meta-analysis of antibody and memory B cells. Vaccine. 2016;34: 1657-64.

25. Muse D, Christensen S, Bhuyan P, et al. A phase 2, randomized, active-controlled, observer-blinded study to assess the immunogenicity, tolerability and safety of bivalent rLP2086, a meningococcal serogroup B vaccine, coadministered with tetanus, diphtheria and acellular pertussis vaccine and serogroup A, C, Y and W-135 meningococcal conjugate vaccine in healthy US adolescents. Pediatr Infect Dis J. 2016;35:673-82.

26. Richmond PC, Marshall HS, Nissen MD, et al. Safety, immunogenicity, and tolerability of meningococcal serogroup B bivalent recombinant lipoprotein 2086 vaccine in healthy adolescents: a randomised, single-blind, placebo-controlled, phase 2 trial. Lancet Infect Dis. 2012;12:597-607.

27. Senders S, Bhuyan P, Jiang Q, et al. Immunogenicity, tolerability, and safety in adolescents of bivalent rLP2086, a meningococcal serogroup B vaccine, coadministered with quadrivalent human papilloma virus vaccine. Pediatr Infect Dis J. 2016;35:548-54.

28. Vesikari T, Ostergaard L, Diez-Domingo J, et al. Meningococcal serogroup B bivalent rLP2086 vaccine elicits broad and robust serum bactericidal responses in healthy adolescents. J Ped Infect Dis Soc. $2015 ; 35: 673-82$.

29. Vesikari T, Wysocki J, Beeslaar J, et al. Immunogenicity, safety, and tolerability of bivalent rLP2086 meningococcal group B vaccine administered concomitantly with diphtheria, tetanus, and acellular pertussis and inactivated poliomyelitis vaccines to healthy adolescents. J Pediatr Infect Dis Soc. 2016;5: 180-7.

30. Ostergaard L, Vesikari T, Absalon J, et al. A bivalent meningococcal B vaccine in adolescents and young adults. N Engl J Med. 2017;377:2349-62.

31. Goldschneider I, Gotschlich EC, Artenstein MS. Human immunity to the meningococcus. I. The role of humoral antibodies. J Exp Med. 1969;129: 1307-26.
32. Borrow R, Balmer P, Miller E. Meningococcal surrogates of protection-serum bactericidal antibody activity. Vaccine. 2005;23:2222-7.

33. McNeil LK, Donald RGK, Gribenko A, et al. Predicting the susceptibility of meningococcal serogroup B isolates to bactericidal antibodies elicited by bivalent rLP2086, a novel prophylactic vaccine. MBio. 2018;9:e00036-18.

34. Jiang HQ, Hoiseth SK, Harris SL, et al. Broad vaccine coverage predicted for a bivalent recombinant factor $\mathrm{H}$ binding protein based vaccine to prevent serogroup B meningococcal disease. Vaccine. 2010;28:6086-93.

35. Belshe RB, Edwards KM, Vesikari $\mathrm{T}$, et al. Live attenuated versus inactivated influenza vaccine in infants and young children. $\mathrm{N}$ Engl J Med. 2007;356:685-96.

36. Monto AS, Ohmit SE, Petrie JG, et al. Comparative efficacy of inactivated and live attenuated influenza vaccines. N Engl J Med. 2009;361:1260-7.

37. Yang Y, Meng Y, Halloran ME, Longini IM Jr. Dependency of vaccine efficacy on preexposure and age: a closer look at a tetravalent dengue vaccine. Clin Infect Dis. 2018;66:178-84.

38. Liko J, Robison SG, Cieslak PR. Pertussis vaccine performance in an epidemic year-Oregon, 2012. Clin Infect Dis. 2014;59:261-3.

39. de Moraes JC, Perkins BA, Camargo MC, et al. Protective efficacy of a serogroup B meningococcal vaccine in Sao Paulo, Brazil. Lancet. 1992;340: 1074-8.

40. Harder T, Koch J, Wichmann O, Hellenbrand W. Predicted vs observed effectiveness of outer membrane vesicle (OMV) vaccines against meningococcal serogroup B disease: systematic review. J Infect. 2017;75:81-94.

41. Engler RJ, Nelson MR, Klote MM, et al. Half- vs fulldose trivalent inactivated influenza vaccine (2004-2005): age, dose, and sex effects on immune responses. Arch Intern Med. 2008;168:2405-14.

42. Bermal N, Szenborn L, Chrobot A, et al. The 10-valent pneumococcal non-typeable Haemophilus influenzae protein $\mathrm{D}$ conjugate vaccine (PHiD-CV) coadministered with DTPw-HBV/Hib and poliovirus vaccines: assessment of immunogenicity. Pediatr Infect Dis J. 2009;28:S89-96.

43. Knuf M, Szenborn L, Moro $\mathrm{M}$, et al. Immunogenicity of routinely used childhood vaccines when coadministered with the 10-valent pneumococcal non-typeable Haemophilus influenzae protein D 
conjugate vaccine (PHiD-CV). Pediatr Infect Dis J. 2009;28:S97-108.

44. Mariani L, Venuti A. HPV vaccine: an overview of immune response, clinical protection, and new approaches for the future. J Transl Med. 2010;8:105.
45. Vidal AC, Smith JS, Valea F, et al. HPV genotypes and cervical intraepithelial neoplasia in a multiethnic cohort in the southeastern USA. Cancer Causes Control. 2014;25:1055-62. 